\title{
Perfect Line Domination in Graphs
}

\author{
N. Pratap Babu Rao \\ Associate Professor \\ S.G. College Koppal 583231 \\ Karnataka, India
}

\begin{abstract}
Here we are defining perfect line domination set and some results on perfect line domination.
\end{abstract}

\section{Keywords}

Line domination, perfect line domination, neighborhood, minimal line domination

\section{INTRODUCTION}

Definition 1. 1 : Perfect line dominating set:

A subset $S$ of $E(G)$ is said to be perfect line dominating set if for each edge not in $S$, e is adjacent to exactly one edge of $S$.

Consider the path $\mathrm{P}_{4}$ with 4 vertices and edge are $\left\{\mathrm{e}_{1}, \mathrm{e}_{2}, \mathrm{e}_{3}\right.$ \}. The set $\left\{\mathrm{e}_{2}\right\}$ is perfect line dominating set in this graph.

It may be noted that If $G$ is a graph then $E(G)$ is always a perfect line dominating set.

Definition 1.2: Minimal perfect line dominating set:

A perfect line dominating set $S$ of the graph $G$ is said to be minimal perfect line dominating set if each line e in $S, S-\{e\}$ is not a perfect line dominating set.

It may be noted that it is not necessary that a proper subset of minimal perfect line dominating set is not a perfect line dominating set

Example 1. 3. Consider the cycle graph $G=C_{5}$ with 5 vertices. Then obviously $\mathrm{E}(\mathrm{G})$ is a minimal perfect line dominating set of $\mathrm{G}$.

However the set $\left\{e_{1}, e_{2}\right\}$ is proper subset of $E(G)$ and is a perfect line dominating set in the graph $\mathrm{G}$.

Definition 1.4: Minimum perfect line dominating set:

A perfect line dominating set with smallest cardinality is called set of the graph minimum perfect line dominating set it is called $\gamma_{\mathrm{pf}}$

Definition1.5: Perfect line domination number:

The cardinality of a minimum perfect line dominating set is called the perfect domination number of the graph $\mathrm{G}$. it is denoted by $\gamma_{\text {pf }}^{\prime}(\mathrm{G})$.

The perfect line domination number of cycle $\mathrm{C}_{6}$ is $2, \mathrm{C}_{5}$ is 2 and that of path $\mathrm{P}_{4}$ is 1 .

Definition 1.6: perfect private line neighborhood:

Let $S$ be the subset of $E(G)$ and e $\epsilon$ S then the perfect private neighborhood of e with respect to $S=P_{p f}[e, S]=\{x \in$ $\mathrm{E}(\mathrm{G})-\mathrm{S} ; \mathrm{N}(\mathrm{x}) \cap\{\mathrm{e}\}\} \cup\{\mathrm{e}$, if e is adjacent to no line of $\mathrm{S}$ or at least line of $\mathrm{S}$.

Theorem 1.7: a perfect line dominating set $S$ of $G$ is minimal perfect line dominating set if and only if for each line e in $S$ $\mathrm{P}_{\mathrm{pf}}[\mathrm{e}, \mathrm{S}]$ is nonempty.
Suppose $\mathrm{S}$ is minimal and $\mathrm{e} \epsilon \mathrm{S}$. Therefore there is a line $\mathrm{x}$ not in $\mathrm{S}-\{\mathrm{e}\}$ or $\mathrm{x}$ is adjacent to at least two lines of $\mathrm{S}-\{\mathrm{e}\}$.

If $\mathrm{x}=\mathrm{e}$ then this implies that $\mathrm{e} \epsilon \mathrm{P}_{\mathrm{pf}}[\mathrm{e}, \mathrm{S}]$

If $x \neq e$ then it is impossible that $x$ is adjacent to at least two lines of $S-\{e\}$. because $S$ is a perfect line dominating set. Therefore $x$ is not adjacent to any line of $S-\{e\}$. Since $S$ is a perfect dominating set $x$ is adjacent to only e in $S$. That is $\mathrm{N}(\mathrm{x}) \cap \mathrm{S}=\{\mathrm{e}\}$ then $\mathrm{x} \in \mathrm{P}_{\mathrm{pf}}[\mathrm{e}, \mathrm{S}]$.

Conversely, suppose $e \epsilon \mathrm{S}$ and $\mathrm{P}_{\mathrm{pf}}[\mathrm{e}, \mathrm{S}]$ contains some line $\mathrm{x}$ of G.

If $x=e$ then $x$ is either adjacent to at least two lines of $S-\{e\}$ Then $S-\{e\}$ is not perfect line dominating set

If $e \neq e$ then $N(x) \cap S=\{e]$ implies that $x$ is not adjacent to any line of $S-\{e\}$.

Thus in all cases $\mathrm{S}-\{\mathrm{e}\}$ is not a perfect line dominating set, if $\mathrm{e} \epsilon \mathrm{S}$. Thus $\mathrm{S}$ is minimal.

Example 1.8. Consider the path $G=P_{5}$ with five lines $e_{1}, e_{2}$, $e_{3}, e_{4} e_{5}$. Note that $S=\left\{e_{2}, e_{4}\right\}$ is minimum and therefore minimal perfect line dominating set $\mathrm{P}_{\mathrm{pf}}\left\{\mathrm{e}_{2}, \mathrm{~S}\right\}=\left\{\mathrm{e}_{1}, \mathrm{e}_{2}\right\}$

We define the following symbols,

$\mathrm{E}_{\mathrm{pf}}^{+}=\left\{\mathrm{e} \in \mathrm{E}(\mathrm{G}) ; \gamma_{\mathrm{pf}}^{\prime}(\mathrm{G}) \leq \gamma_{\mathrm{pf}}^{\prime}(\mathrm{G}-\mathrm{e})\right\}$

$\mathrm{E}_{\mathrm{pf}}^{-}=\left\{\mathrm{e} \in \mathrm{E}(\mathrm{G}) ; \gamma_{\mathrm{pf}}^{\prime}(\mathrm{G})>\gamma_{\mathrm{pf}}^{\prime}(\mathrm{G}-\mathrm{e})\right\}$

$\mathrm{E}_{\mathrm{pf}}^{0}=\left\{\mathrm{e} \in \mathrm{E}(\mathrm{G}) ; \gamma_{\mathrm{pf}}^{\prime}(\mathrm{G})=\gamma_{\mathrm{pf}}^{\prime}(\mathrm{G}-\mathrm{e})\right\}$

Remark; The above sets are mutually disjoint and their union is $E(G)$

Lemma; 1. 9. Let $e \in E(G)$ and suppose e is a pendent line and has a neighbor $\mathrm{x}$ if $\mathrm{e} \in \mathrm{E}_{\mathrm{pf}}^{-}$then

$$
\gamma_{\mathrm{pf}}^{\prime}(\mathrm{G}-\mathrm{e})=\gamma_{\mathrm{pf}}^{\prime}(\mathrm{G})-1 \text {. }
$$

Let $S_{1}$ be a minimum line dominating set of

$E-\{e\}$ if $x \in S_{1}$ then $S_{1}$ is a perfect line dominating set of $G$ with $\left|\mathrm{S}_{1}\right|<\gamma_{\mathrm{pf}}(\mathrm{G})$

i.e., $\gamma_{\mathrm{pf}}^{\prime}(\mathrm{G}) \leq\left|\mathrm{S}_{1}\right| \leq \gamma_{\mathrm{pf}}^{\prime}(\mathrm{G}-\mathrm{e})$ this is a contradiction, therefore $\quad \mathrm{x} \notin \mathrm{S}_{1}$ let $\mathrm{S}=\mathrm{S}_{1} \cup\{\mathrm{x}\}$. Then $\mathrm{S}$ is minimum perfect line dominating set of G. Therefore $\gamma_{\mathrm{pf}}^{\prime}(\mathrm{G})=|\mathrm{S}|=$ $\left|\mathrm{S}_{1}\right|+1=\gamma_{\mathrm{pf}}^{\prime}(\mathrm{G}-\mathrm{e})+1$

Hence the lemma.

Theorem 1.10. Let e e a line of $G$ Then $e \in \mathrm{E}_{\mathrm{pf}}^{+}$if and only if the following conditions are satisfies, i) e belongs to every $\gamma_{\text {pf }}^{\prime}$ set of G, (ii) no subset $\mathrm{s}$ of $\mathrm{G}-\{\mathrm{e}]$ which is either disjoint from $\mathrm{N}[\mathrm{e}]$ or intersects $\mathrm{N}[\mathrm{e}]$ in at least two linesand $|\mathrm{S}| \leq \gamma_{\mathrm{pf}}$ can be perfectly dominating set of 
$\mathrm{G}-\{\mathrm{e}\}$.

Proof: (i) Suppose $e \in \mathrm{E}_{\mathrm{pf}}^{+}$,

Suppose $\mathrm{S}$ is a $\gamma_{\mathrm{pf}}^{\prime}$ set of $\mathrm{G}$ which does not contains e then $\mathrm{S}$ is a perfect dominating set of

$\mathrm{G}-\{\mathrm{e}\}$.

Therefore $\gamma_{\mathrm{pf}}^{\prime}(\mathrm{G}-\mathrm{e}) \leq|\mathrm{S}|=\gamma_{\mathrm{pf}}^{\prime}(\mathrm{G})$. thus e $\notin \mathrm{E}_{\mathrm{pf}}^{+}$. This is a contradiction. Then e must belong to every $\gamma_{\mathrm{pf}}$ set of G,

(ii) If there is set $\mathrm{S}$ which satisfies the condition stated in (ii) then $\mathrm{S}$ is a perfect line dominating set of $\mathrm{G}-\{\mathrm{e}]$ and therefore $\gamma_{\mathrm{pf}}^{\prime}(\mathrm{G}-\mathrm{e}) \leq \gamma_{\mathrm{pf}}^{\prime}(\mathrm{G})$ This is a contradiction.

Conversely, assume that (i) and (ii) hold

Suppose e $\in \mathrm{E}_{\mathrm{pf}}^{0}$. Let $\mathrm{s}$ be a minimum perfect line dominating set of $\mathrm{G}-\{\mathrm{e}\}$.

Then $|\mathrm{S}|=\gamma_{\mathrm{pf}}^{\prime}(\mathrm{G})$.

Suppose e is not adjacent to any line of $\mathrm{S}$. Then $\mathrm{S}$ is disjoin from $\mathrm{N}[\mathrm{e}] .|\mathrm{S}|=\gamma_{\mathrm{pf}}(\mathrm{G})$. and $\mathrm{S}$ is perfectly line dominating set of $\mathrm{G}-\{\mathrm{e}\}$. This violates (ii)

Suppose e is adjacent to exactly one line of $S$ then $S$ is minimum perfect line dominating set of $\mathrm{G}$ not containing e which violates (i)
Suppose e is adjacent to at least two lines of $S$, then $|S| \cap N[e]$ in at least two lines and $\mathrm{S}$ is perfectly line dominating set of $\mathrm{G}$ $-\{\mathrm{e}]$ with $|\mathrm{S}|=\gamma_{\mathrm{pf}}^{\prime}(\mathrm{G})$ which again violates (ii).

Thus $\mathrm{e} \in \mathrm{E}_{\mathrm{pf}}^{0}$ implies (i) and (ii) violated.

\section{ACKNOWLEDGEMENTS}

We would like to express our thanks the referee, for their careful reading and valuable suggestions

\section{REFERENCES}

[1] Acharya BD 1980 "The strong domination number of graph and related concepts. J.maths.phy.Sci vol14 no.5 pp471-475.

[2] Berge C 1973 Graphs and Hyper graphs, North-Holland Amsterdam

[3] Jayaram S.R. Line domination in graphs-Line domination in graphs, graphs and combinotorics vol3 Dec1987 pp357-363

[4] Muthulakshmi.T 2014 'Theory-Domination related parameters in graph $\mathrm{PhD}$ thesis Anna University Chennai.

[5] Nordhus E.A and Gaddum J.W 1956 'On Complimentary graphs Amer. Math. Monthly vol63 pp175-177. 